\title{
Attenuation of vasospasm and hemoglobin-induced constriction in the rabbit basilar artery by a novel protease inhibitor
}

\author{
Barbara Cappelletto, M.D., Hakan H. Caner, M.D., Frank Schottler, Ph.D., Aij-Lie Kwan, M.D., \\ David Eveleth, Ph.D., Patricia L. Foley, D.V.M., Neal F. Kassell, M.D., and Kevin S. Lee, Ph.D. \\ Department of Neurological Surgery, University of Virginia Health Sciences Center, Charlottesville, \\ Virginia; and Cortex Pharmaceuticals, Inc., Irvine, California
}

Calcium-activated proteolysis mediated by the protease inhibitor, calpain, has recently been implicated in the pathogenesis of cerebral vasospasm. The effect of one inhibitor of calcium-activated proteolysis, z-Leu-Phe-CONH-morpholene (zLF), on cerebrovascular constriction was examined in two experimental paradigms. In the first paradigm, the rabbit basilar artery (BA) was visualized via a transclival exposure, and its diameter was monitored using videomicroscopy. In the second experimental paradigm two intracisternal injections of autologous blood were administered to mimic a subarachnoid hemorrhage $(\mathrm{SAH})$. The BA was visualized via the transclival exposure, and its luminal diameter was measured. Topical application of oxyhemoglobin $(\mathrm{OxyHb})$, a known pathogenic agent in cerebral vasospasm, elicited vasoconstriction in normal animals, reducing arterial diameter to approximately $75 \%$ of resting levels. Pretreatment with zLF $(100,200$, or $300 \mu \mathrm{M})$ attenuated vasoconstriction induced by OxyHb. In an experimental model of SAH, the diameter of the BA was reduced after the first injection of blood to approximately $67 \%$ of normal resting levels when measured 3 to 4 days later. This vasospastic response was reversed significantly by topical application of zLF $(100 \mu \mathrm{M})$; vascular diameter was increased to approximately $84 \%$ of normal resting levels.

These findings demonstrate that both acute OxyHb-induced constriction and blood-induced vasospasm are sensitive to an inhibitor of the proteolytic enzyme, calpain. Together, these observations indicate an important role for calcium-activated proteolysis in the development and maintenance of vasospasm after SAH. In addition, it may be inferred from the data that inhibitors of calcium-activated proteolysis may be useful therapeutic agents for treating this form of cerebrovascular disease.

\section{Key Words * vasospasm * calpain * hemoglobin * basilar artery * rabbit}

The mechanisms underlying subarachnoid hemorrhage (SAH)-induced vasospasm remain a matter of some discussion. Substantial evidence has accrued indicating that hemoglobin $(\mathrm{Hb})$ is a primary pathogenic agent in this vasospastic response;[11] however, the potential mechanisms responsible for the spastic constrictor response to blood or $\mathrm{Hb}$ are controversial. One recent hypothesis concerning the development of vasospasm suggests that a limited proteolysis of protein kinase (PK) $\mathrm{C}$ occurs in vascular smooth muscle following $\mathrm{SAH}$ and that this response is mediated by the $\mathrm{Ca}^{++}$-activated protease 
calpain.[17] According to this hypothesis, a limited proteolysis by calpain converts PKC to PKM,[8,19] thus enhancing kinase activity and leading to vasoconstriction. There are several attractive features of this hypothesis for describing SAH-induced vasospasm. First, an appropriate signal (that is, elevated $\left[\mathrm{Ca}^{++}\right]_{\mathrm{i}}$ ) is responsible for triggering the response. Hemoglobin has been shown to elicit increases in the intracellular levels of $\mathrm{Ca}^{++}$in vascular smooth muscle,[26,27,32] and this increase could trigger a proteolytic response. Second, $\mathrm{Ca}^{++}$-dependent activation of calpain results in the proteolysis of critical substrate proteins, [8,31] such as PKC, that could directly contribute to vasoconstriction.[13,14] Finally, excessive activation of calpain could lead to sustained constriction that would be difficult to reverse using conventional receptor agonists or antagonists. An autolytic modification of calpain was recently demonstrated in spastic canine arteries after experimental SAH;[37] the autolytic response results in a functional activation of calpain caused by an irreversible reduction in its requirement for $\mathrm{Ca}^{++} .[5,25]$ Subsequent to a change of this type, even small increases in $\left[\mathrm{Ca}^{++}\right]_{i}$ could be capable of activating the protease after $\mathrm{SAH}$; this effect could contribute to a constrictor response that is not easily reversed.

In the present study the hypothesis that $\mathrm{Ca}^{++}$-activated proteolysis participates in cerebral vasospasm was examined by testing the effects of a protease inhibitor that is both potent and relatively specific for calpain. The protease inhibitor, z-Leu-Phe-CONH-morpholene (zLF), was selected from a series of structurally related compounds[10] based on its efficacy in intact cell systems, as well as its specificity, potency, and solubility. Results derived from the data presented here demonstrate the utility of this compound for blocking vasoconstriction elicited by oxyhemoglobin $(\mathrm{OxyHb})$ and for reversing established vasospasm following SAH.

\section{MATERIALS AND METHODS}

\section{Characteristics of the Protease Inhibitor zLF}

The inhibitor zLF is a peptide ketoamide inhibitor of calpain, the structure of which is shown in Fig. 1. The interaction of this class of inhibitors with calpains has been described in detail elsewhere.[10] Briefly, the peptide ketoamides are reversible transition-state inhibitors that form hemithioketal enzyme-inhibitor complexes on reaction with the active cysteine site of calpains. As determined by the methods described elsewhere,[10] zLF has an inhibition constant $\left(\mathrm{k}_{\mathrm{i}}\right)$ of $1100 \mathrm{nM}$ for human calpain I and $\mathrm{a} \mathrm{k}_{\mathrm{i}}$ of $330 \mathrm{nM}$ for rabbit calpain II. It inhibits other thiol proteases such as cathepsin B and papain much less efficiently than it does the calpains $\left(\mathrm{k}_{\mathrm{i}}=13,000 \mathrm{nM}\right.$ and $195,000 \mathrm{nM}$, respectively).

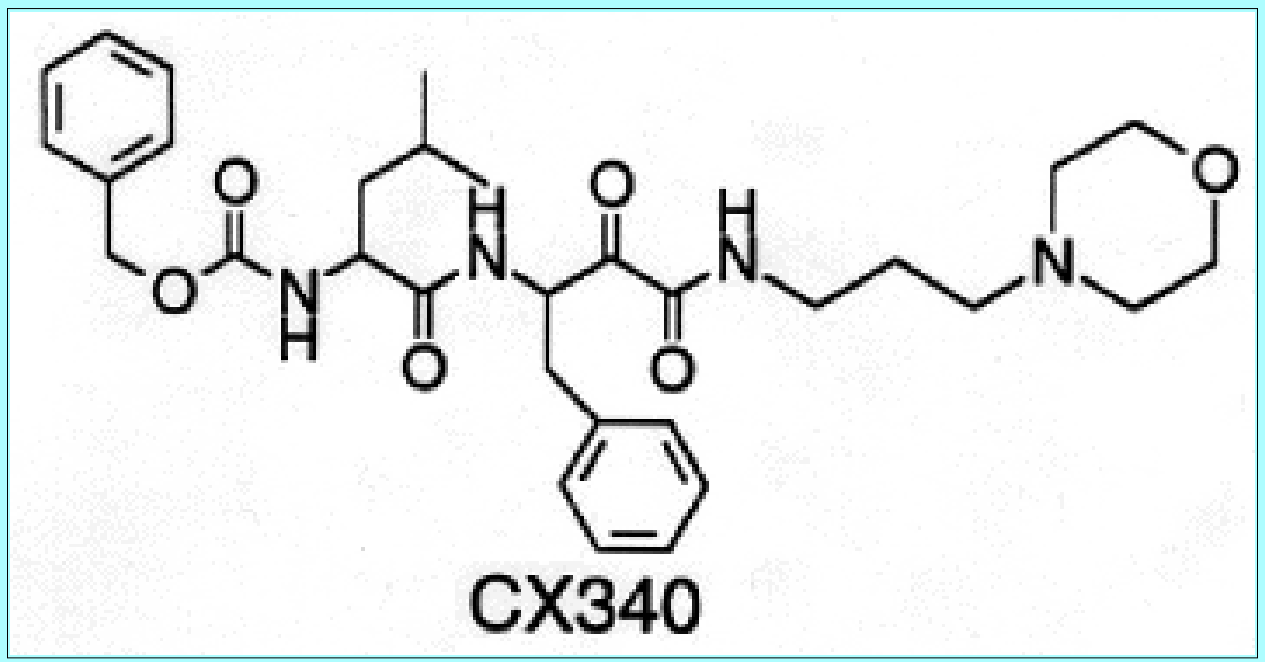


Fig. 1. Illustration showing the chemical structure of the calpain inhibitor zLF.

\section{Acute Oxyhemoglobin Treatment}

New Zealand White male rabbits, weighing between 2.5 and $3.5 \mathrm{~kg}$, were used in experiments examining the effects of zLF on OxyHb-induced constriction. All procedures on rabbits described here and in subsequent experiments were conducted in accordance with guidelines approved by the University of Virginia Animal Research Committee.

Rabbits were anesthetized with an intramuscular injection of ketamine $(50 \mathrm{mg} / \mathrm{kg})$ and xylazine $(8$ $\mathrm{mg} / \mathrm{kg}$ ). Anesthesia was supplemented as necessary during the remainder of the experiment. After inducing anesthesia, the animals were intubated and paralyzed by an intramuscular injection of pancuronium bromide $(0.2 \mathrm{mg} / \mathrm{kg})$; paralysis was maintained by repeated administration of $0.2 \mathrm{mg} / \mathrm{kg}$ every 30 minutes. Animals were ventilated with a small-animal ventilator. The right femoral artery and vein were cannulated to monitor blood pressure, withdraw arterial blood, and maintain hydration with intravenous lactated Ringer's solution. The arterial blood pressure and heart rate were monitored continuously by a pressure transducer. The arterial $\mathrm{pH}, \mathrm{PaCO}_{2}$, and $\mathrm{PaO}_{2}$ were measured every 40 minutes and maintained within physiological ranges.

The basilar artery (BA) was exposed via a transclival approach.[7] The muscles were coagulated, and the edges of the clival window were sealed with bone wax to keep the field free of blood. Before opening the dura mater, a needle was placed on one side of the bone window to permit superfusion of artificial cerebrospinal fluid (aCSF). The aCSF was composed of the following (in $\mathrm{mM}$ ): $124 \mathrm{NaCl}, 3.3 \mathrm{KCl}, 1.25$ $\mathrm{KH}_{2} \mathrm{PO}_{4}, 2.4 \mathrm{MgSO}_{4}, 2.0 \mathrm{CaCl}_{2}, 25.7 \mathrm{NaHCO}_{3}$, and 10 glucose (pH 7.3). The artery was superfused continuously with aCSF maintained at $37 \mathrm{~s} \mathrm{C}$ to ensure a stable temperature and to prevent exposure to ambient air. A needle connected to a suction apparatus was positioned on the opposite side of the bone window to maintain a constant fluid level. After opening the dura mater, the arachnoid was gently removed. Occasional mechanical stimulation of the BA during opening resulted in transient, segmental constriction, as described previously; [7] the experiment was resumed following resolution of these brief constrictions. In three cases, the BA was exposed to excessive hemorrhage, at which point the experiment was terminated.

The BA was visualized by using a stereomicroscope fitted with a charge-coupled device camera. The image was projected onto a videomonitor, and the arterial diameter was measured using a digital caliper system. Arterial diameter, blood pressure, and heart rate were measured and recorded every 5 minutes. A superfusion rate of $1 \mathrm{ml} /$ minute over the BA was maintained with aCSF for at least 15 minutes while stable baseline measurements were obtained. Animals were then treated with: 1) aCSF alone, 2) aCSF + vehicle, or 3$)$ aCSF + zLF $(100,200$, or $300 \mu \mathrm{M})$ superfused at a rate of $1 \mathrm{ml} /$ minute. The vehicle solution in these experiments was standard aCSF containing 0.15 to $0.32 \%$ acetic acid (pH 7.3). The preparation was treated with these solutions for at least 20 minutes, and $\mathrm{OxyHb}\left(10^{-4} \mathrm{M}\right)$ was then added for 15 to 80 minutes, after which aCSF alone was reintroduced.

\section{SUBARACHNOID HEMORRHAGE MODEL}

A model of SAH was used in which two injections of autologous blood were administered into the cisterna magna of the 27 rabbits. Anesthesia was induced and ventilation was maintained as described in the acute study (see previous section). The central ear artery was cannulated with a 22-gauge Teflon catheter to obtain autologous blood. A 23-gauge butterfly needle was inserted percutaneously into the 
cisterna magna, and $1.5 \mathrm{ml} / \mathrm{kg}$ of autologous arterial blood was injected over a period of 20 seconds. The animals were placed in a head-down position for 15 minutes and were extubated when fully awake. Two days later, the rabbits were reanesthetized, and the procedure was repeated with $1 \mathrm{ml} / \mathrm{kg}$ of blood. Three to 4 days after the first injection, the BA was exposed via the transclival approach described previously. A small portion of the blood clot around the BA was carefully removed, avoiding mechanical stimulation of the artery. The artery was superfused for up to 55 minutes with aCSF alone, after which zLF (100 $\mu \mathrm{M})$, vehicle, or aCSF was added for up to 2 hours. Arterial diameter, blood pressure, $\mathrm{pH}, \mathrm{PaO}_{2}$, and $\mathrm{PaCO}_{2}$ were monitored as described previously. Three rabbits that showed severe neurological signs (ataxia, hemiplegia) following SAH were excluded from the study, and three animals were excluded because of an absence of arterial spasm.

\section{Preparation of Solutions}

Oxyhemoglobin solutions were prepared as described elsewhere.[12] Briefly, rabbit $\mathrm{Hb}$ was dissolved in distilled water, and sodium dithionite was used to reduce the levels of methemoglobin. The solution was dialyzed twice against distilled water at $4 \check{\mathrm{s} C}$ for 2 hours. The relative amounts of $\mathrm{OxyHb}$ and methemoglobin were then determined by spectroscopy.[27] Solutions containing at least $70 \%$ OxyHb were selected for use, and aliquots were frozen and stored at $-80 \mathrm{~s} \mathrm{C}$ for a maximum of 3 days.

The zLF was dissolved in distilled water and acetic acid. This stock solution was diluted with aCSF to obtain working solutions of 100,200 , or $300 \mu \mathrm{M}$ zLF. The final concentration of acetic acid in the working solutions ranged from 0.15 to $0.32 \%$. The $\mathrm{pH}$ was adjusted to 7.3 with $1 \mathrm{~N} \mathrm{NaOH}$. At a concentration of $300 \mu \mathrm{M}, \mathrm{zLF}$ was near saturation in the aCSF solution.

\section{Statistical Analysis}

Values given for the diameter of the BA are means \pm standard errors calculated for each animal derived from individual measurements made every 5 minutes, or from 10- to 15 -minute averages obtained at various time points during the different treatments. Unless otherwise noted, these data are expressed as a percentage of the average diameter measured during the last 15 minutes of the pretreatment baseline period.

Analysis of variance and Bonferroni post-hoc tests were used to examine group effects at different time points after treatment with $\mathrm{OxyHb}$ in the acute experiments. The same tests were used to examine group effects in vessels from post-SAH animals treated with $\mathrm{zLF}$, aCSF alone, or vehicle. A paired t-test was used to evaluate the effects of vehicle or zLF on baseline responses and on the recovery after OxyHb-induced constriction in the acute study.

\section{Sources of Supplies and Equipment}

The zLF (CX340) was provided by Cortex Pharmaceuticals, Inc., Irvine, CA, and the rat Hb was obtained from Sigma Chemical Co., St. Louis, MO.

The small-animal ventilator (model 683) was obtained from Harvard Apparatus, Inc., S. Natick, MA. The pressure transducer (model 78342A) was purchased from Hewlett-Packard, Palo Alto, CA. The Wild M8 stereomicroscope was purchased from Bunton Instrument Co., Rockville, MD; the charge-coupled device camera was supplied by World Video Inc., Boyerton, PA; and the digital caliper system (model 601 HM) by Stahl Research Lab., Inc., Port Chester, NY. 


\section{RESULTS}

\section{Effect of zLF on Acute Treatment With Oxyhemoglobin}

Topical application of $\mathrm{OxyHb}\left(10^{-4} \mathrm{M}\right)$ to the transclivally exposed BA in animals without SAH elicited a constriction that was stable during the entire treatment period. When treated with OxyHb alone, vessels constricted in seven animals to $74.3 \pm 3.3 \%$ of baseline diameter after 15 to 20 minutes of treatment and to $76.3 \pm 2.9 \%$ in five animals after 35 to 40 minutes of treatment. This effect was reversed after reintroduction of aCSF alone. Figure 2 shows examples of the responses of $\mathrm{BAs}$ to $\mathrm{OxyHb}$ alone and to OxyHb in the presence of vehicle or zLF (100-300 $\mu \mathrm{M})$. Pretreatment of the arteries with zLF substantially reduced the amount of constriction elicited by OxyHb. Significant group differences were found in comparisons of averages taken at 15 to 20 minutes $\left(F_{4,27}=15.1, p<0.001\right)$ and at 35 to 40 minutes $\left(\mathrm{F}_{4,21}=21.1, \mathrm{p}<0.001\right)$. Oxyhemoglobin-induced constriction was significantly reduced in the zLF-treated vessels (100, 200, and $300 \mu \mathrm{M}$; Fig. 2 lower) when compared with arteries pretreated with vehicle or aCSF alone ( $\mathrm{p}<0.006$ and $\mathrm{p}<0.001$ for all comparisons at 15-20 minutes and 35-40 minutes, respectively; Bonferroni post-hoc tests). The amount of constriction observed in the $\mathrm{OxyHb}+$ vehicle group at 15 to 20 minutes ( $78.4 \pm 1.6 \%, 12$ animals) and 35 to 40 minutes ( $77 \pm 1.5 \%$, nine animals) was not significantly different from the constriction observed in the OxyHb alone group. 

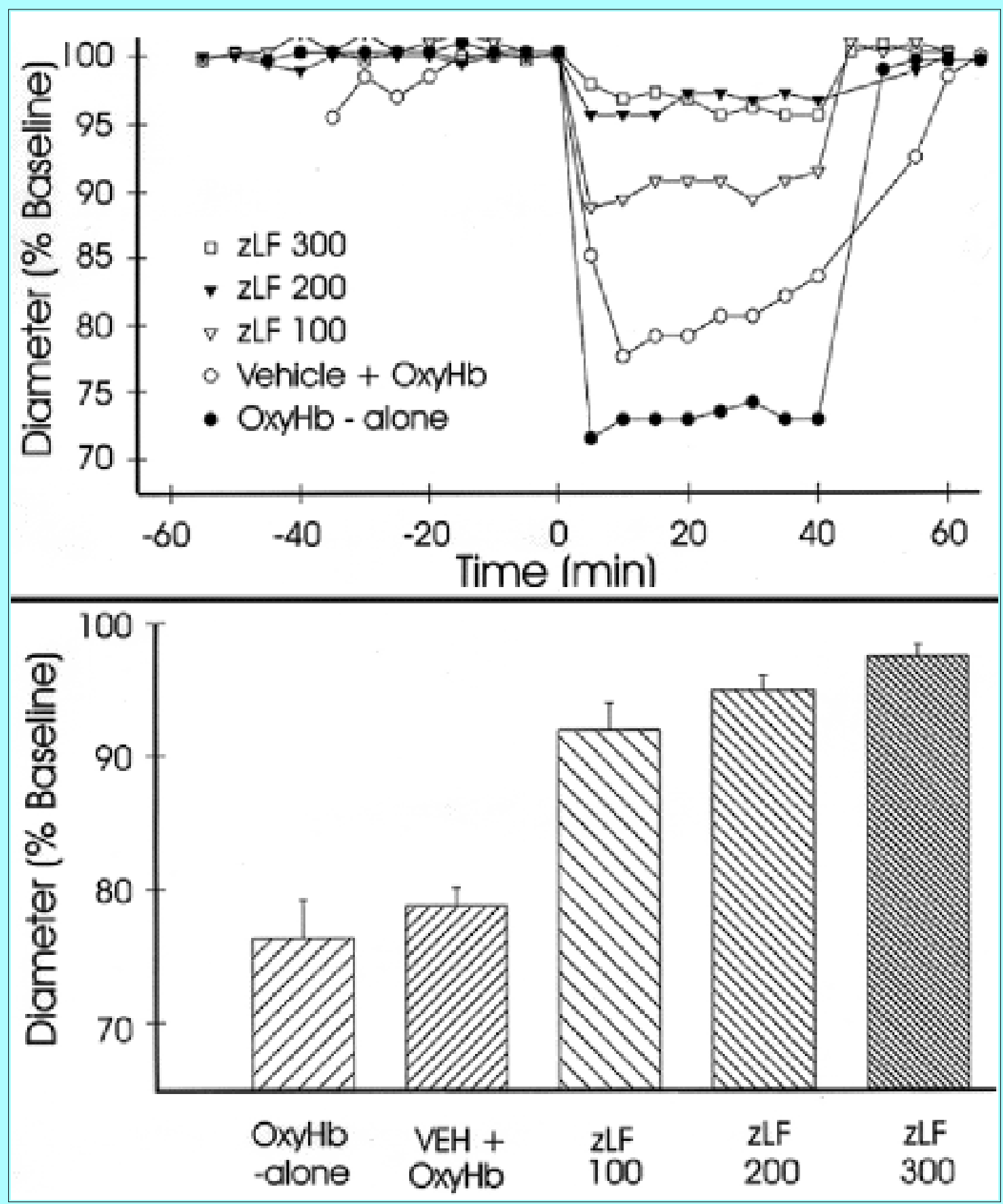

Fig. 2. Graphs showing the effect of zLF on acute application of OxyHb. Upper: Scatterplot showing the average diameter of the BA 35 to 40 minutes after treatment with OxyHb. Constrictions elicited by OxyHb in the presence of 100,200 , or $300 \mu \mathrm{M}$ zLF were reduced significantly ( $\mathrm{p}<0.001$ for all comparisons, Bonferroni post-hoc tests) relative to arteries superfused with aCSF (OxyHb alone) or vehicle ( $\mathrm{VEH}+\mathrm{OxyHb})$. Constrictions in the $\mathrm{OxyHb}$ alone and $\mathrm{VEH}+\mathrm{OxyHb}$ groups did not differ from one another. The diameters of the BAs are expressed as percentages of the values measured during the last 10 minutes of the predrug baseline period. Time 0 marks the onset of a 40 -minute infusion of $\mathrm{OxyHb}\left(10^{-4}\right.$ $\mathrm{M})$. Lower: Bar graph illustrating constrictions of the BA in response to treatment with $\mathrm{OxyHb}$ alone, OxyHb in the presence of vehicle (vehicle $+\mathrm{OxyHb}$ ), or OxyHb in the presence of various concentrations $(100,200$, and $300 \mu \mathrm{M})$ of zLF.

The effect of zLF appeared to be dose dependent across the range of concentrations tested; however, no significant differences were found among the different doses of zLF (Bonferroni post-hoc tests). In 
animals pretreated with $100 \mu \mathrm{M} z \mathrm{LF}$, the diameter of BAs was reduced to $90.5 \pm 1.8 \%$ (seven animals) and $91.8 \pm 2.1 \%$ (six animals) at 15 to 20 and 35 to 40 minutes, respectively, after the addition of $\mathrm{OxyHb}$. In contrast, a near-complete blockade of OxyHb-induced constriction was observed in experiments in which $300 \mu \mathrm{M}$ OxyHb was used; the diameter of the BAs was $98.6 \pm 1.1 \%$ and $97.4 \pm$ $0.9 \%$ at similar time points (three animals). Values for OxyHb-induced constriction in animals pretreated with $200 \mu \mathrm{M}$ zLF were intermediate to those obtained with $100 \mu \mathrm{M}$ and $300 \mu \mathrm{M}$ zLF $(93.8 \pm 1.3 \%$ at $15-20$ minutes [three animals] and $94.9 \pm 1.1 \%$ at $35-40$ minutes [three animals]).

\section{Effect of Vehicle or Drug on Baseline Diameter}

Neither the vehicle (acetic acid) nor zLF affected the resting diameter of BAs in normal (non-SAH) animals. The diameters of BAs were $99.6 \pm 0.8 \%, 100.2 \pm 0.2 \%$, and $100.2 \pm 0.2 \%$ of predrug levels at 20 to 60 minutes after treatment with 100, 200, or $300 \mu \mathrm{M}$ zLF, respectively. The mean BA diameter was $99.9 \pm 0.4 \%$ in the vehicle-treated group at a similar time.

\section{Effect of zLF on Vasospasm Following SAH}

In the second set of experiments we injected autologous blood into the cisterna magna to mimic SAH. Basilar arteries were examined via the transclival approach 3 to 4 days after the first injection of blood. This protocol necessitated partial clot removal to visualize the arteries during the transclival surgery. In seven cases, this procedure significantly altered or affected the stability of the constricted BA, and these animals were excluded from the study. Stable SAH-induced constriction, varying less than $15 \%$ during the pretreatment baseline period, was observed in 14 animals. The BAs in these 14 animals were constricted to an average diameter of $552 \pm 18 \mu \mathrm{m}$; this value corresponds to $67 \%$ of the resting diameter observed in vessels in all non-SAH animals described in the preceding section.

Topical application of zLF dilated the constricted BAs (Fig. 3). Analysis of variance revealed that the diameter of the BA, expressed as a percentage of the pretreatment diameter, was significantly different among groups after 45 to 60 minutes of treatment $\left(F_{2,11}=25.4 ; p<0.001\right)$. In seven animals, BAs treated with zLF dilated to $127.4 \pm 3.3 \%$ of their predrug (constricted) baselines, and they were significantly larger in comparison to both aCSF-treated $(103.7 \pm 0.9 \%, \mathrm{p}<0.001$; three animals) and vehicle-treated $(101.3 \pm 1.2 \%, \mathrm{p}<0.001$; four animals) vessels at this time (Bonferroni post-hoc tests). The diameters of vehicle- and aCSF-treated arteries were stable and did not differ significantly from one another or from their earlier baseline values. 


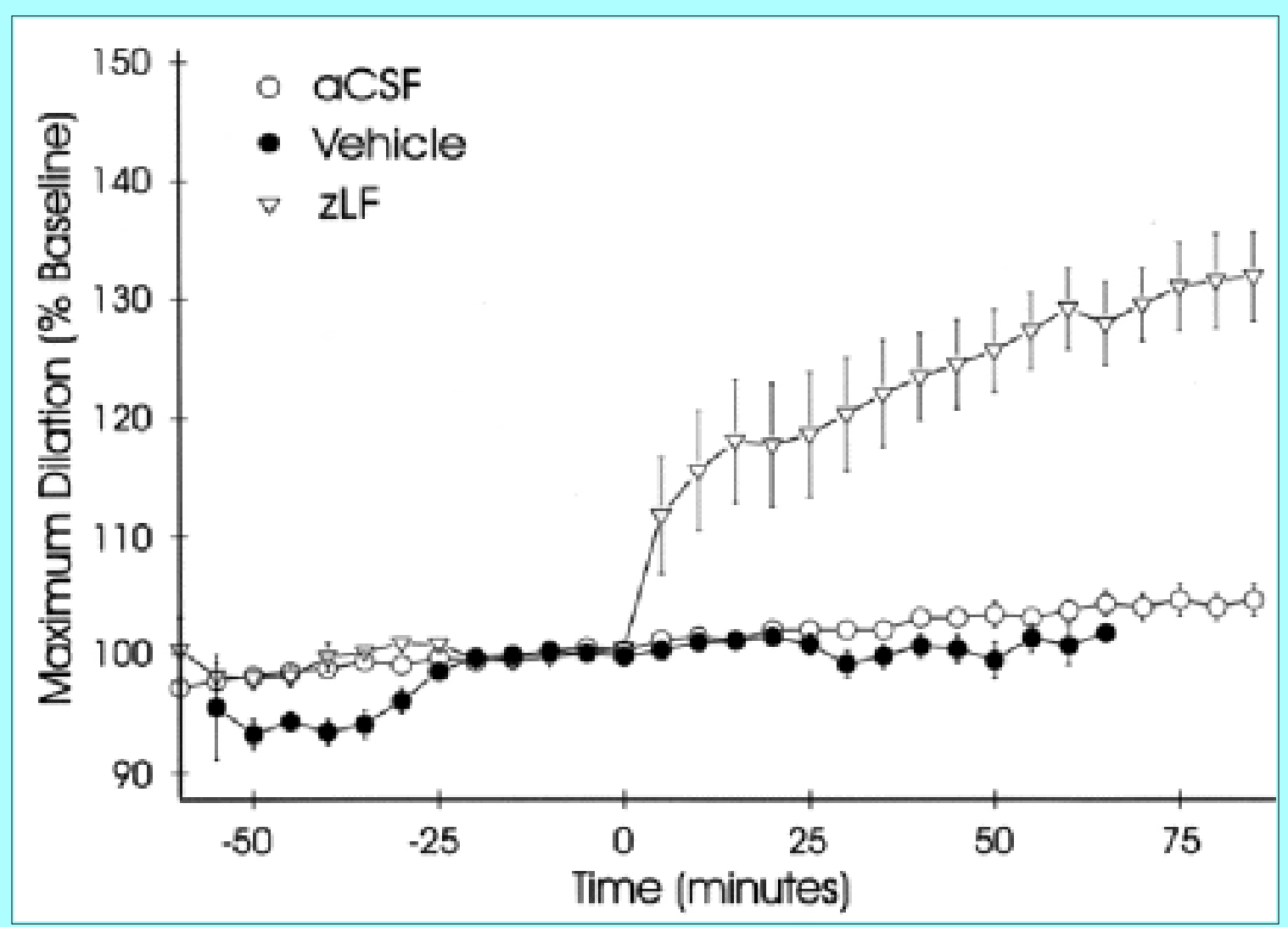

Fig. 3. Scatterplot showing the effects of $100 \mu \mathrm{M}$ zLF on the diameter of spastic BAs in animals subjected to SAH. The diameter of the arteries was measured at 5-minute intervals; values are expressed as percentages of the values measured during the last 10 minutes of the pretreatment baseline period. Time 0 marks the onset of treatment with vehicle or $100 \mu \mathrm{M}$ zLF; the nontreated group (aCSF only) is also shown. Treatment with zLF produced a dilation of BAs preconstricted by SAH.

In some cases, zLF-treated vessels continued to dilate for up to 2 hours before achieving a stable dilation during treatment. The maximum dilation of zLF-treated arteries correlated well with the magnitude of the clot-induced constriction observed at the start of the experiment. This relationship was found whether the magnitude of the dilation was expressed as a percentage of pretreatment baseline periods $(r=0.95)$ or in absolute units (micrometers; $r=0.77$ ). The average maximum amount of dilation in zLF-treated arteries was $167 \pm 10 \mu \mathrm{m}$. Treatment with zLF increased the diameter of SAH-constricted arteries from $523 \pm 24$ $\mu \mathrm{m}$ to a stable, maximum value of $690 \pm 18 \mu \mathrm{m}$ (that is, 132\%). When expressed as a percentage of non-SAH control values, the spastic arteries dilated to a maximum level of $84 \%$ after prolonged treatment with zLF.

\section{DISCUSSION}

The absence of an effective clinical treatment for reversing pathological vasoconstriction following aneurysmal SAH continues to encourage the investigation of mechanisms involved in blood-induced vasoconstriction. Oxyhemoglobin is widely believed to be a central pathogenic agent contributing to cerebral vasospasm.[11,15,20] However, the potential mechanisms in vascular wall constituents through which various forms of $\mathrm{Hb}$ may elicit this type of vascular constriction are numerous and remain a matter of discussion.[4,11,29,30,35] In the present study we examined two aspects of this issue: first, the participation of $\mathrm{Ca}^{++}$-activated proteolysis in the initial phase of OxyHb-induced constriction was evaluated by pretreating vessels with a calpain inhibitor; and second, the contribution of $\mathrm{Ca}^{++}$-activated 
proteolysis to delayed cerebral vasospasm was examined by treating spastic vessels with the calpain inhibitor.

Vasoconstriction induced by the acute application of OxyHb was inhibited by pretreatment with zLF. Oxyhemoglobin-induced constriction was virtually eliminated in the presence of $300 \mu \mathrm{M} z \mathrm{LF}$. It thus appears that the initial constrictor response to $\mathrm{OxyHb}$ requires proteolytic activation for its expression. The proteolytic response to OxyHb could presumably occur at the level of the smooth muscle and/or endothelium, inasmuch as OxyHb-induced effects on both of these vascular wall constituents have been implicated in cerebral vasospasm. [3,11,30] The roles played by calpain in different vascular wall constituents continue to be elucidated in ongoing investigations.

The ability of zLF, a relatively specific inhibitor of calpain, to inhibit the acute response to OxyHb suggests that $\mathrm{Ca}^{++}$-activated proteolysis is an integral component of the initial contractile response after $\mathrm{SAH}$. Indeed, evidence has recently begun to appear supporting the hypothesis that calpain is activated after SAH. First, a preferred substrate for calpain, spectrin, has been shown to be degraded in a rabbit model of experimental SAH.[9] Second, levels of the native form of calpain are reduced following SAH in the same model, suggesting that an autolytic conversion of calpain to its activated form has occurred.[37] Finally, levels of the autolytically activated form of calpain are increased in a canine model of SAH.[37] It can be inferred from these data indicate that calpain is functionally activated following SAH.

Calpain has several potential substrates in vascular smooth muscle that could contribute to sustained vasoconstriction. Several of these substrates are listed in Fig. 4, and their potential roles in vasospasm are discussed in the following sections. One prominent substrate of calpain is PKC. In the BA, stimulation of PKC elicits a constriction that is both pronounced and sustained.[22,24] Limited proteolysis of PKC by calpain produces an activated variant of PKC: PKM, which exhibits a reduced $\mathrm{Ca}^{++}$requirement for its activation.[19] Limited proteolysis of PKC by calpain could therefore enhance kinase activity, leading to constriction. The participation of activated PKC in cerebral vasospasm is also consistent with observations from studies in which kinase inhibitors were used. In a canine model of SAH, vasospasm is sensitive to a general kinase inhibitor (H-7), which can inhibit the catalytic subunit of PKC,[14,17] but it is insensitive to an inhibitor of the regulatory subunit of PKC, calphostin C.[17] In addition, levels of the native form of PKC in the cytosol are reduced in conjunction with vasospasm, [28] an effect that would be expected subsequent to proteolysis. An activation of PKC via proteolytic dissociation of its catalytic and regulatory subunits is consistent with these sets of observations. 


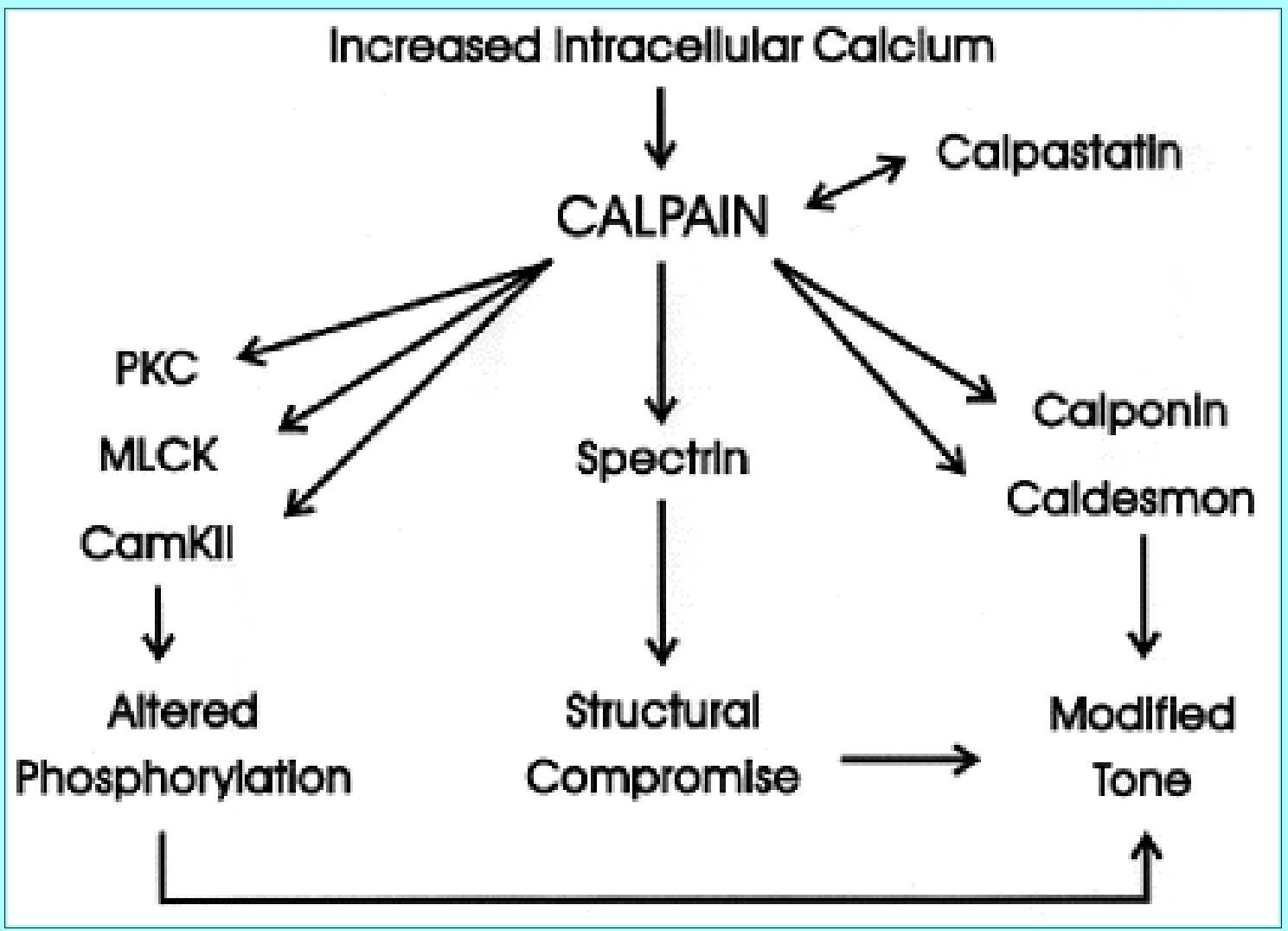

Fig. 4. Schematic illustration of calpain substrates in smooth muscle and their potential role in vasospasm. Calpain has several intracellular substrates in smooth muscle whose degradation could serve as a pathophysiological mechanism of cerebral vasospasm. Several of the possible roles calpain-mediated proteolysis could play in modifying cerebrovascular tone are depicted.

Despite increasing evidence implicating PKC in vasospastic constriction, caution should be maintained in interpreting the available evidence. The loss of cytosolic activity of an enzyme during a pathological response does not establish a causal link between the enzyme and response. Moreover, in a recent study it has been suggested that there are discrepancies between arterial PKC activity and the extent of arterial contraction; it may be inferred from the data that vasospasm cannot be explained solely by the activation of PKC.[22] Another important issue when evaluating the role of PKC in vasospasm is the interpretation of studies in which kinase inhibitors are used to block vasoconstriction. The kinase inhibitors used in previous studies of vasospasm exhibited only limited specificity.[6] For instance, inhibitors that interfere with the interaction of adenosine triphosphate (ATP) and protein substrate with the catalytic domain of PKC (such as H-7) are also effective against other kinases. This lack of specificity is due to the conservation of the ATP-binding site in many kinases.[6] Caution must be exercised in the interpretation of studies in which multifunctional inhibitors are used, such as H-7. The precise role played by PKC in the setting of vasospasm awaits further experimental clarification.

Myosin light chain kinase (MLCK) is another calpain substrate[31] that could contribute to the constrictor response. Partial proteolysis of MLCK reduces its requirements for $\mathrm{Ca}^{++}$and calmodulin, thus enhancing MLCK activity. Increased phosphorylation of the myosin light chain, which is well correlated with the development of force during phasic contraction, $[1,18,21,23]$ could then result in vasoconstriction. Direct evidence demonstrating kinase modification by calpain following SAH has yet to be presented. Nonetheless, kinase activation, be it PKC, MLCK, or some other kinase, represents a plausible mechanism through which calpain could mediate the constrictor response to OxyHb. 
Calponin and caldesmon are two other contraction-related substrates for calpain[31] that are also capable of modulating vascular tone.[2,33,36] These thin filament-associated proteins inhibit actomyosin ATPase and appear to assist in maintaining smooth muscle in a relaxed state. Although the effect of calpain-mediated proteolysis on these substrates in the BA is unclear, it is possible that a substantial proteolytic response could alter the inhibitory influence of these proteins on vasoconstriction.

In addition to its direct effects on smooth-muscle contraction, $\mathrm{Ca}^{++}$-activated proteolysis could also contribute to morphological modifications of the BA. Several cytoskeletal proteins are preferred substrates for calpain, and one such substrate, spectrin, has been shown to be degraded after experimental SAH in the rabbit.[9] A disturbance in the cytoskeleton of endothelial or smooth-muscle cells could contribute to the various structural and functional derangements that accompany the later stages of vasospasm.

The results of the present study demonstrate that cerebral vasospasm elicited by experimental SAH can be substantially reversed by zLF. At a concentration of $100 \mu \mathrm{M}$, zLF dilated vessels to $132 \%$ of their constricted levels after SAH. Only a single dosage of zLF $(100 \mu \mathrm{M})$ was tested in these experiments because of the limited availability of the compound; it therefore remains unclear whether a maximum effect was achieved. Nonetheless, these results demonstrate that spastic constriction of the BA is amenable to treatment with a calpain inhibitor, a finding that is consistent with a recent report.[17]

It can be inferred from the data presented here that $\mathrm{Ca}^{++}$-activated proteolysis is involved in both the early and late phases of the vascular response to blood constituents. A prolonged effect of calpain following SAH is plausible and could be due to: 1) continuous activation of the protease by high $\mathrm{Ca}^{++}$ levels; 2) stable conversion of calpain to its autolyzed form, which has a reduced requirement for $\mathrm{Ca}^{++}$; and/or 3) a reduction in the activity of endogenous inhibitory mechanisms that usually limit enzymatic activity. If the autolyzed form of calpain remains stable for sustained periods in the vascular wall, then a brief period of autolysis could result in the protracted activation of, and lowered $\mathrm{Ca}^{++}$requirement for, calpain. Evidence confirms that autolytic activation of calpain occurs in the canine BA after SAH. In addition, this group of investigators has demonstrated a reduction in the levels of calpastatin, an endogenous enzyme that normally serves to inhibit calpain activity.[37] These findings indicate that at least two regulatory mechanisms that restrict calpain activation are attenuated after SAH. Important goals of future studies will be to define the type(s) of calpain involved in vasoconstrictor responses and to clarify the roles of their modified regulatory systems.

\section{CONCLUSIONS}

A calpain inhibitor was shown to substantially reduce vasoconstriction induced by $\mathrm{OxyHb}$ and to reverse partially established vasospasm induced by the injection of blood. The current observations support the hypothesis that $\mathrm{Ca}^{++}$-activated proteolysis is an important feature of these pathological forms of constriction. Although the precise substrates through which calpain modifies vascular tone remain unknown, the latest generation of protease inhibitors[10,16,34] may provide important tools for limiting the deleterious effects of cerebral vasospasm.

\section{Acknowledgments}

We thank Dr. Oren Sagher for his critical reading of this work and Mrs. Sarah B. Hudson for assistance in the preparation of this manuscript. 


\section{References}

1. Aksoy MO, Murphy RA, Kamm KE: Role of $\mathrm{Ca}^{++}$and myosin light chain phosphorylation in regulation of smooth muscle. Am J Physiol 242:C109-C116, 1982

2. Chalovich JM: Actin mediated regulation of muscle contraction. Pharmacol Ther 55:95-148, 1992

3. Comair YG, Schipper HM, Brem S: The prevention of oxyhemoglobin-induced endothelial and smooth muscle cytoskeletal injury by deferoxamine. Neurosurgery 32:58-65, 1993

4. Fujiwara S, Kassell NF, Sasaki T, et al: Selective hemoglobin inhibition of endothelium-dependent vasodilation of rabbit basilar artery. J Neurosurg 64:445-452, 1986

5. Goll DE, Thompson VF, Taylor RG, et al: Is calpain activity regulated by membranes and autolysis or by calcium and calpastatin? BioEssays 14:549-556, 1992

6. Huang KP: The mechanism of protein kinase C activation. Trends Neurosci 12:425-432, 1989

7. Kapp J, Mahaley MS Jr, Odom GL: Cerebral arterial spasm. Part 1: Evaluation of experimental variables affecting the diameter of the exposed basilar artery. J Neurosurg 29:331-338, 1968

8. Kishimoto A: Limited proteolysis of protein kinase C by calpain, its possible implication. Adv Second Messenger Phosphoprotein Res 24:472-477, 1990

9. Lee KS, Foley PL, Vanderklish P, et al: The role of calcium-activated proteolysis in vasospasm after subarachnoid hemorrage, in Findlay JM (ed): Cerebral Vasospasm. Amsterdam: Elsevier, 1993, pp $85-88$

10. Li Z, Patil GS, Golubski ZE, et al: Peptide alpha-keto ester, alpha-keto amide, and alpha-keto acid inhibitors of calpains and other cysteine proteases. Am Chem Soc 36:3472-3480, 1993

11. Macdonald RL, Weir B: A review of hemoglobin and the pathogenesis of cerebral vasospasm. Stroke 22:971-982, 1991

12. Martin W, Villani GM, Jothianandan D, et al: Selective blockade of endothelium-dependent and glyceryl trinitrate-induced relaxation by hemoglobin and by methylene blue in the rabbit aorta. $\mathbf{J}$ Pharmacol Exper Ther 232:708-716, 1985

13. Matsui T, Sugawa M, Johshita H, et al: Activation of the protein kinase C-mediated contractile system in canine basilar artery undergoing chronic vasospasm. Stroke 22:1183-1187, 1991

14. Matsui T, Takuwa Y, Johshita H, et al: Possible role of protein kinase C-dependent smooth muscle contraction in the pathogenesis of chronic cerebral vasospasm. J Cereb Blood Flow Metab 11:143-149, 1991

15. Mayberg MR, Okada T, Bark DH: Morphologic changes in cerebral arteries after subarachnoid hemorrage. Neurosurg Clin North Am 1:417-432, 1990

16. Mehdi S: Cell-penetrating inhibitors of calpain. Trends Biochem Sci 164:150-153, 1991

17. Minami N, Tani E, Maeda Y, et al: Effects of inhibitors of protein kinase $\mathrm{C}$ and calpain in 
experimental delayed cerebral vasospasm. J Neurosurg 76:111-118, 1992

18. Murphy RA, Aksoy MO, Dillon PF, et al: The role of myosin light chain phosphorylation in regulation of the cross-bridge cycle. Fed Proc 42:51-56, 1983

19. Murray AW, Fournier A, Hardy SJ: Proteolytic activation of protein kinase C: a physiological reaction? Trends Biochem Sci 12:53-54, 1987

20. Osaka K: Prolonged vasospasm produced by the breakdown products of erythrocytes. J Neurosurg 47:403-411, 1977

21. Sakaki S, Ohue S, Khono K, et al: Impairment of vascular reactivity and changes in intracellular calcium and calmodulin levels of smooth muscle cells in canine basilar arteries after subarachnoid hemorrage. Neurosurgery 25:753-761, 1989

22. Sako M, Nishihara J, Ohta S, et al: Role of protein kinase C in the pathogenesis of cerebral vasospasm after subarachnoid hemorrage. J Cereb Blood Flow Metab 13:247-254, 1993

23. Stull JT, Bowman BF, Gallagher PJ, et al: Myosin phosphorylation in smooth and skeletal muscles: regulation and function. Prog Clin Biol Res 327:107-126, 1990

24. Sugawa M, Koide T, Naitoh S, et al: Phorbol 12,13-diacetate-induced contraction of the canine basilar artery: role of protein kinase C. J Cereb Blood Flow Metab 11:135-142, 1991

25. Suzuki K, Imajoh S, Emori Y, et al: Calcium-activated neutral protease and its endogenous inhibitor. Activation at the cell membrane and biological function. FEBS Lett 220:271-277, 1987

26. Takanashi Y, Weir BKA, Vollrath B, et al: Time course of changes in concentration of intracellular free calcium in cultured cerebrovascular smooth muscle cells exposed to oxyhemoglobin. Neurosurgery 30:346-350, 1992

27. Takenaka K, Yamada H, Sakai N, et al: Cytosolic calcium changes in cultured rat aortic smooth-muscle cells induced by oxyhemoglobin. J Neurosurg 74:620-624, 1991

28. Takuwa Y, Matsui T, Abe Y, et al: Alterations in protein kinase $\mathrm{C}$ activity and membrane lipid metabolism in cerebral vasospasm after subarachnoid hemorrhage. J Cereb Blood Flow Metab 13:409-415, 1993

29. Toda N, Kawakami M, Yoshida K: Constrictor action of oxyhemoglobin in monkey and dog basilar arteries in vivo and in vitro. Am J Physiol 260:H420-H425, 1991

30. Tsuji T, Weir BK, Cook DA: Time-dependent effects of extraluminally-applied oxyhemoglobin and endothelial removal on vasodilator responses in isolated, perfused canine basilar arteries. Pharmacology 38:101-112, 1989

31. Tsunekawa S, Takahashi K, Abe M, et al: Calpain proteolysis of free and bound forms of calponin, a troponin T-like protein in smooth muscle. FEBS Lett 250:493-496, 1989

32. Vollrath B, Weir BKA, Macdonald RL, et al: Intracellular mechanisms involved in the responses of cerebrovascular smooth-muscle cells to hemoglobin. J Neurosurg 80:261-268, 1994

33. Walsh MP: Smooth muscle caldesmon. Prog Clin Biol Res 327:127-140, 1990 
34. Wang KK: Developing selective inhibitors of calpain. Trends Pharmacol Sci 11:139-142, 1990

35. Wellum GR, Irvine TW Jr, Zervas NT: Cerebral vasoactivity of heme proteins in vitro. Some mechanistic considerations. J Neurosurg 56:777-783, 1982

36. Winder SJ, Walsh MP: Smooth muscle calponin. Inhibition of actomyosin MgATPase and regulation by phosphorylation. J Biol Chem 265:10148-10155, 1990

37. Yamaura I, Tani E, Saido T, et al: Calpain-calpastatin system of canine basilar artery in vasospasm. J Neurosurg 79:537-543, 1993

Manuscript received August 18, 1997.

Accepted in final form September 17, 1997.

This research was supported by National Institutes of Health Grant No. HL49396 and by a grant from Cortex Pharmaceuticals, Inc., to Dr. Lee.

Address reprint requests to: Kevin S. Lee, Ph.D., Department of Neurological Surgery, University of Virginia Health Sciences Center, Box 420, Charlottesville, Virginia 22908. 